\title{
Review of: "Effects of Surgical Facemasks on Perceived Exertion During Submaximal Exercise in Healthy Children"
}

simon driver, Katelyn Brown

Potential competing interests: The author(s) declared that no potential competing interests exist.

Journal Information

Journal Name

Research Square

Manuscript Title

Effects of Surgical Facemasks on Perceived Exertion During Submaximal Exercise in Healthy Children Manuscript Type

Preprint

Summary

Review by a native English speaker will increase readability of this article. These authors present an investigation into the impact of surgical face masks in children 8-12 on RPE, dyspnea, HR, SpO2, and performance on a short submaximal sit to stand test. The authors cite literature regarding the effects of N95 masks in children of the same age group, which did not have significant impact on SpO2 and HR during activity, so justification on why the authors think surgical masks would impair these parameters would strengthen this paper. Authors also need to explain their choice to dichotomize the age groups and why they chose to exclude overweight children. The tools chosen are appropriate for this age group. The results section is very unclear. Define "clinical relevance" much earlier in the text.

Abstract

The authors use the term "wearability" but none of the outcomes address this construct (fit, breathability, etc.). Language should reflect the actual outcomes assessed.

Specify that you used a surgical mask rather than "facemask" throughout the entire document.

Consider listing variables measured instead of "cardiorespiratory response" (SpO2 and HR).

Describe sample sex

Clarify what your results are describing (can't tell which are FM and which are control)/

Why did you dichotomize age group?

Clinically relevant? Describe.

Were your results statistically significant? Just state "it was higher with the face mask".

Explain how you measured "submaximal performance". Repetitions?

Again, would consider listing variables rather than using cardiorespiratory demand. 
You mention that wearing the surgical mask did not impact performance but also indicated that RPE was higher in the masked condition - while the difference was non-significant this indicates there was an impact. The authors need to clarify this language.

Introduction

Prior to reference 7, use another word other than "solve".

Explain what findings you think are similar to adults. RPE?

There are several recent scientific papers that examined the impact of facemasks on adult exercise performance. These should be mentioned rather than the generic WHO guideline currently referenced

Materials and Methods: Subjects

Provide justification for excluding overweight children and children at risk for being overweight Materials and Methods: Outcomes

So, participants completed 3 tests - each an hour apart? Trial - test 1- test 2 ? Was the trial completed without a mask?

Description of when facemask was applied is unclear.

Suggest specifying "RPE" defined by modified Borg and refer to RPE for the rest of the paper.

$1^{\prime}$ is this one minute?

Explain your method for measuring cardiorespiratory demand

Why should the STST "be considered as submaximal effort and it reflects the activities of daily living."? The authors should provide a rationale.

What length of time was HR measured? (15 seconds? 30 seconds? 1 minute?

Why was a change of 1.1SD used for the sample size calculation? Results

Provide reference for your choice for $80 \%$ as submax rather than $85 \%$

Standardize your variables for primary outcome (RPE, perceived exertion, mBorgf)

Clarify which outcomes for RPE were face mask and control. You state the order as (face mask and control) and state that the face mask was higher, but that is not how the numerical results read.

Explain clinical relevance or did you mean statistically significant?

"HR adaptation" is not what you are measuring as this term refers to change over time, consider using HR response.

No need to say "cardiac demand" just state HR.

Discussion

Define "clinically relevant" much earlier in the text.

"Smaller effort" to "reduced"

Table

Description of age in header and in first row is redundant

Consider table categories as Baseline, Peak and Recovery 
